\title{
The Evaluation of Expanded Uncertainty of DC Voltages in the Presence of Electromagnetic Interferences using the LabVIEW Environment
}

\author{
Przemysław Otomański ${ }^{1}$, Eligiusz Pawłowski ${ }^{2}$, Anna Szlachta ${ }^{3}$ \\ ${ }^{1}$ Institute of Electrical Engineering and Electronics, Faculty of Control, Robotics and Electrical Engineering, Poznan \\ University of Technology, Piotrowo street 3A, 60-965 Poznań, Poland, przemyslaw.otomanski@put.poznan.pl \\ ${ }^{2}$ Department of Automation and Metrology, Faculty of Electrical Engineering and Computer Science, Lublin University of \\ Technology, Nadbystrzycka street 38A, 20-618 Lublin, Poland \\ ${ }^{3}$ Department of Metrology and Measurement Systems, Faculty of Electrical and Computer Engineering, Rzeszow University \\ of Technology, W. Pola street 2, Rzeszow, 35-959, Poland
}

\begin{abstract}
The paper presents a possible application of integrated LabVIEW environment to the final evaluation of measurement results in direct measurement. The possibilities of presenting and visualizing the uncertainty of measurement results in a convenient and user-friendly form are also discussed. The topics discussed in the paper were presented using a developed application in LabVIEW. The paper discusses the topic of measurement of direct voltages in the presence of strong electromagnetic interferences. These problems are frequently omitted or hardly emphasized. It presents a suitable measuring system, a virtual measuring instrument created in the LabVIEW environment, and the results of tests carried out for an example NI PCI-6221 data acquisition board. The described approach can be applied also in other measurement situations.
\end{abstract}

Keywords: Expanded uncertainty, probability distribution, virtual instrument, electromagnetic interference.

\section{INTRODUCTION}

In industrial conditions, measuring systems can be exposed to various interferences. Interferences that infiltrate into the measuring system are the source of additional measurement errors, which increase the uncertainties of the final measurement result.

In general, the electrical interferences can be classified into a few types, depending on its coupling mechanism. Without losing generality, four types of interference can be considered due to the path of propagation: electromagnetic radiation, capacitive cross-talk, inductive cross-talk, and conductive cross-talk by common ground wire.

Infiltration of interferences through an electromagnetic wave occurs when an appliance as a source of radio waves is located near the measuring system. The conductive components of the measuring system act as antennae, in which currents being the source of measurement errors are induced.

The electromagnetic disturbances are also a problem for designers of electronic circuits and systems. With the miniaturization of electronic circuits, an increase in the probability of interference occurring can be observed [1].
Capacitive cross-talk is a situation in which an alternative voltage on one line can cause an interference on an adjacent line because of the capacitance between the lines. In this case, any AC signal in one line will induce voltage in the nearby capacitively coupled line.

Interferences infiltrating through magnetic coupling occur when a conductor providing alternating current is near the measuring system forming a closed loop. An alternating magnetic field is formed around such a conductor and by infiltrating into the measuring circuit it induces a current as a source of errors. This effect is compounded if there are ferromagnetic components nearby, which form a magnetic circuit increasing this impact.

Conducted interferences occur in systems in which the measuring circuit's ground points that are distant from each other have different potentials. This causes a flow of equalizing currents in the ground conductors and the formation of additional voltage decreases that sum up with the signal being measured.

Conducted interferences are observed in electrical equipment during its operation, e.g. when switching off receivers, the fast electric current pulses arise due to self- 
induction (bursts), when switching inductive or capacitive receivers on and off in the supply system. In addition, they arise during lightning discharges, electrostatic discharges, power loss, transients, voltage drops, flicker, and emission of harmonic currents.

There are methods to limit the level of electrical interferences, e.g. by their reducing (shielding, grounding, galvanic insulation, using filters and separations and other ways of limiting - cutting the amplitude, dissipating part of the energy), and also by their limiting to a "safe" level of interaction with the sensitive devices [2]. All the abovementioned types of interferences reduce the quality of the measurement system by reducing accuracy. For low signal levels, the impact of noise will be significant. In the presented work, the radiated disturbances caused by interferences infiltrating through magnetic coupling are analyzed in relation to the standard IEC/EN 61000-4-8 [3]. The main aim of the work is to assess the uncertainty of the measurement system in the presence of electromagnetic disturbances using the LabVIEW environment. There are many methods of evaluating the inaccuracy of the measurement results, presented in the papers of many authors [4], [5], [6]. For this purpose, a specially developed virtual instrument was used and it is described in the paper.

\section{EXPERIMENTAL RESEARCH}

The diagram of the measuring system applied in the tests is presented in Fig.1. It is composed of a personal computer (PC) with an installed PCI 6221 data acquisition board (DAQ) by National Instruments and with the LabVIEW environment, of a REF5050 [7] low-noise, low-drift, very high precision and stable source of reference voltage $V_{R E F}$, and of a ferromagnetic core FC ensuring the magnetic coupling of the measuring circuit with the source of interferences being the L-N-PE line powering the computer. The REF5050 is a low-noise ( $3 \mu \mathrm{VPP} / \mathrm{V})$, low-temperature drift $\left(3 \mathrm{ppm} /{ }^{\circ} \mathrm{C}\right)$, very high accuracy $(0.05 \%)$ voltage reference [7]. These features make the REF5050 ideal for use in high-precision data acquisition systems. As a result, the influence of the reference voltage on the DAQ uncertainty estimation is negligible.

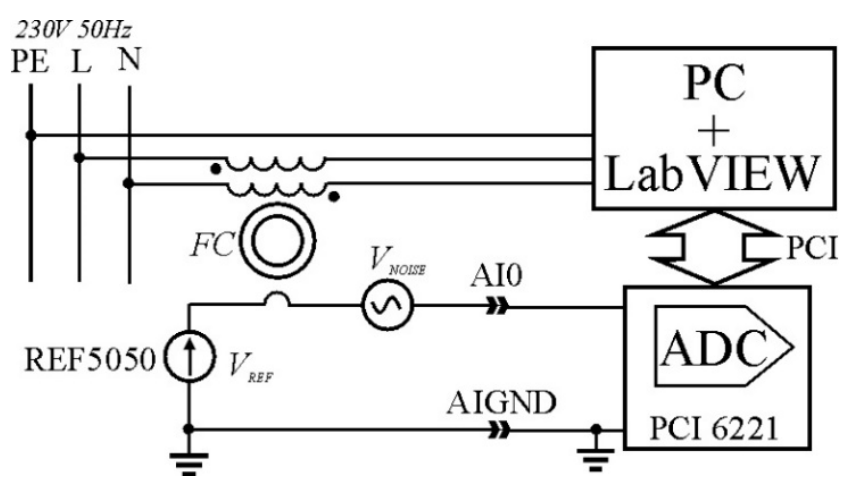

Fig.1. Diagram of the measuring system applied in the tests.

The PC is powered from the $230 \mathrm{~V} 50 \mathrm{~Hz}$ grid through a system that ensures a magnetic coupling with the measuring circuit. The L-N powering conductors are coiled on the toroidal ferromagnetic core FC in such a manner that the created magnetic fluxes sum up. A conductor as a component of the measuring system can be passed through the hole in the FC. The current powering the computer contains a range of higher harmonics being a source of strong interferences, which infiltrate into the measuring system through magnetic coupling in the form of interference voltage $V_{\text {NOISE }}$. The DAQ is connected to the computer via the PCI bus. A source of stable reference voltage $V_{R E F}=+5 \mathrm{~V}$ was connected to the AI0 analog input of the DAQ. Measurements with a low level of interferences require placing the conductors that form the measuring circuit at least $1 \mathrm{~m}$ away from the conductors that power the computer. Measurements with a high level of interferences are achieved by passing the measuring circuit conductor through the hole in the FC. This causes an increase in the level of interferences in the form of a circa 300-times increase in the type A uncertainty.

\section{UNCERTAINTY OF MEASUREMENT}

The uncertainty of measurement result is a parameter allowing to determine the limits of an interval containing with assumed probability - an unknown value of the true measurand.

The cause of the uncertainty of measurement result is the fact that we do not know the exact value of the measurand [8]. This is because the results obtained in the process of direct measurement reveal errors in both the uncertainties of type A, which are the result of random effects, and the uncertainties of type B, caused by systematic effects. According to the recommendations of an international document [8], about the method of evaluating and expressing uncertainty, the following notations and symbols will be adopted, corresponding to the parameters of probability distributions [9]: - standard uncertainty of type A, calculated on the basis of observed scatter of the results of a series of measurements, which is equal to the estimator of the standard deviation for average:

$$
u_{A}=\sqrt{\frac{1}{n(n-1)} \sum_{i=1}^{n}\left(x_{i}-\bar{x}\right)^{2}}=\bar{S}_{\bar{x}}
$$

- standard uncertainty of type B, equal to the standard deviation of the assumed distribution of apparatus errors. With the assumption that the apparatus errors have rectangular distribution within the limits of maximum error $\pm \Delta_{g}:$

$$
u_{B}=\frac{\Delta_{g}}{\sqrt{3}}=\sigma_{J}
$$

- combined standard uncertainty for a directly measured value, when the standard uncertainties of type A and type B are taken into consideration:

$$
u_{\mathrm{c}}=\sqrt{u_{A}^{2}+u_{B}^{2}}
$$


- expanded uncertainty:

$$
U=k(\alpha) \cdot u_{c}
$$

- $k(\alpha)$ is the coverage factor, corresponding to the standardized variable of a given distribution.

\section{UNCERTAINTY BUDGET}

Final measurement result is complete only when it contains both the measurand value and the measurement uncertainty attributed to that value.

Statement of all identified component standard uncertainties estimated with A and B type methods is called uncertainty budget [9]. The aim of creating the uncertainty budget is to prove that the combined uncertainty of measurement result $u_{c}$ was estimated in a matter-of-fact, penetrating and verifiable way. With a correctly constructed budget, we can prove that all elements of uncertainty have been analyzed, also those that were not the greatest parts of the combined uncertainty. It means that no element was rejected arbitrarily, based on beliefs or traditional manner of conduct [10]. The subject of uncertainty budget evaluation is discussed in many publications, for example in [11], [12].

\section{VIRTUAL INSTRUMENT}

LabVIEW (Laboratory Virtual Instrument Engineering Workbench) is a graphical programming software used in developing programs for simulation, data acquisition, control, and communication application. In the LabVIEW environment icons are interconnected to create a program generally referred to as a VI (Virtual Instrument) [13], [14], [15]. All VIs must have two components: the Front Panel and the Block Diagram. The Front Panel contains various controls and indicators while the Block Diagram includes a variety of functions. The functions (icons) are wired inside the Block Diagram, where the wires represent the flow of data. The execution of a VI is data dependent, which means that a node inside the Block Diagram will execute only if data is available at each input terminal of that node. By contrast, the execution of a traditional program, such as $\mathrm{C}$ program, follows the order in which the instructions are written [16]. VIs make up a new generation of measurement equipment. In these new devices the hardware realization of some functions is replaced with an appropriate program executed by a PC. It concerns mainly the device handling and the execution of signal processing algorithms. Thanks to those facilities, it is easy to modify such a device and execute the signal processing algorithms. In particular, the LabVIEW environment seems to be commonly used at the university level teaching process for realization of specific programs for presentation of various problems.

In practice, virtual measuring instruments are often used for determining the value of measurement errors. Examples of such instruments are presented in the papers [17], [18]. For the purpose of determining errors and uncertainties of measurements in the system shown in Fig.1., a virtual instrument was prepared in the LabVIEW environment.
Fig.2. shows a portion of the application interface that displays the configuration parameters of the data acquisition board.

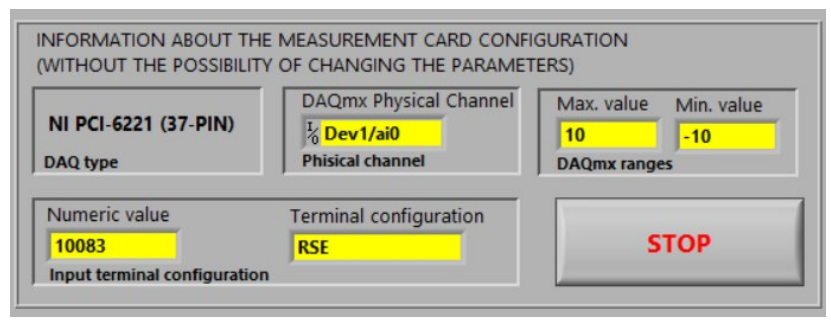

Fig.2. Configuration parameters of the data acquisition board.

The developed VI application enables DC voltage measurements in a series with a preset number of $n$ samples. Table 1. presents selected parameters of the DAQ card used.

Table 1. AI Absolute accuracy of the DAQ used [19].

\begin{tabular}{|c|c|c|c|c|}
\hline $\begin{array}{c}\text { Nominal Range Positive } \\
\text { Full Scale }\end{array}$ & 10 & 5 & 1 & 0.2 \\
\hline $\begin{array}{c}\text { Nominal Range Negative } \\
\text { Full Scale }\end{array}$ & -10 & -5 & -1 & -0.2 \\
\hline $\begin{array}{c}\text { Residual Gain Error } \\
\text { (ppm of Reading) }\end{array}$ & 75 & 85 & 95 & 135 \\
\hline $\begin{array}{c}\text { Residual Offset Error } \\
\text { (ppm of Range) }\end{array}$ & 20 & 20 & 25 & 80 \\
\hline $\begin{array}{c}\text { Offset Tempco } \\
\left.\text { (ppm of Range/ }{ }^{\circ} \mathrm{C}\right)\end{array}$ & 57 & 60 & 79 & 175 \\
\hline $\begin{array}{c}\text { Random Noise, } \\
\text { ( } \mu \mathrm{Vrms})\end{array}$ & 244 & 122 & 30 & 13 \\
\hline $\begin{array}{c}\text { Absolute Accuracy at Full } \\
\text { Scale }(\mu \mathrm{V})\end{array}$ & 3100 & 1620 & 360 & 112 \\
\hline Sensitivity $(\mu \mathrm{V})$ & 97.6 & 48.8 & 12.0 & 5.2 \\
\hline$\quad$
\end{tabular}

Actual values of the completed measurements are indicated in the upper part of Fig.3.

The data enabling the calculation of the measurement uncertainties are entered into the software in the bottom part of Fig.3. in the green windows. All measurements were made in the $10 \mathrm{~V}$ Full Scale Range. As we can see in Table 1., on this range, the parameters involved in the error budget formation are as follows: Residual Gain Error $=75 \mathrm{ppm}$ of Reading, Residual Offset Error $=20$ ppm of Range, Offset Tempco $=57 \mathrm{ppm}$ of Range $/{ }^{\circ} \mathrm{C}$, Random Noise $=244 \mu \mathrm{Vrms}$. Additionally, in Table 1., the manufacturer specifies the sensitivity and the absolute accuracy of the DAQ card. Both of these parameters have no influence on the estimation of uncertainty. According to the M Series Data Acquisition Device Specifications, the sensitivity is the smallest voltage change that can be detected. It is a function of noise. Absolute accuracy at full scale on the analog input channels is determined using the following assumptions: Reading equal to Range, Number of Readings $=100$, Coverage Factor $=3$. Full scale absolute accuracy is worth comparing with 
experimentally determined measurement uncertainty. It is also worth noting that the accuracy is significantly inferior to the sensitivity at all ranges.

The created application enables measurements of direct voltage in a series of a preset number $n$ of samples, calculates the average value $\bar{x}$, the measurement uncertainties $u_{A}, u_{B}$, $u_{c}, U$, the board's maximum error $\Delta_{g}$, and performs an additional statistical analysis of results [20].

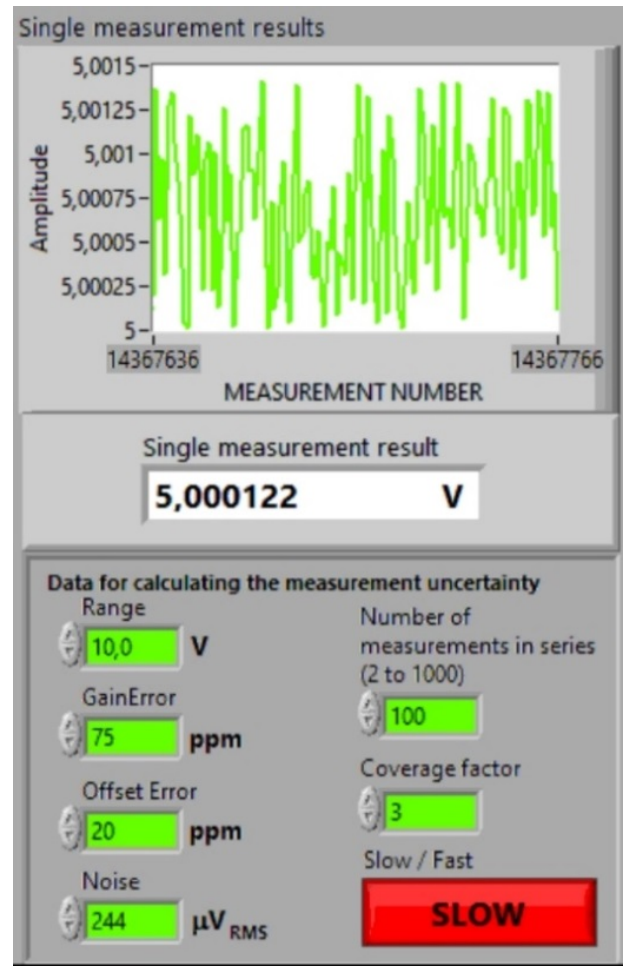

Fig.3. Single measurement results and absolute accuracy of DAQ.

Fig.4. shows the series of measurements used at the moment for averaging and determining all the other parameters is indicated.

The data enabling the calculation of the measurement uncertainties are entered into the software in the left bottom part of the panel in the green windows. The calculation results are indicated next to them in the middle part of the panel: average value (red field), uncertainty of type A (blue field), uncertainty of type $\mathrm{B}$, combined uncertainty and maximum error of the ADC (pink color), and expanded uncertainty (purple color). The right part of the panel contains a chart presenting averaged results of measurements from successive series and an additional statistical analysis of the results. The number of measurement series $N$, which will be subjected to an additional analysis, can be entered in the green field.

In Fig.5., average values of successive series of measurements are marked red in the chart, the range with the width of $\pm U$ is marked purple, and the range with the width of $\pm 3 \sigma$ is marked blue.

Above all, the standard deviation for a single result $s\left(x_{i}\right)$ and the standard deviation of the average value $s(\bar{x})$ from successive measurement series are calculated. Theoretically, the ratio of these two values should be equal to the square root of $n$ of the averaged measurements.

The software makes it possible to verify this theoretical correlation based on measurements carried out in an actual system.

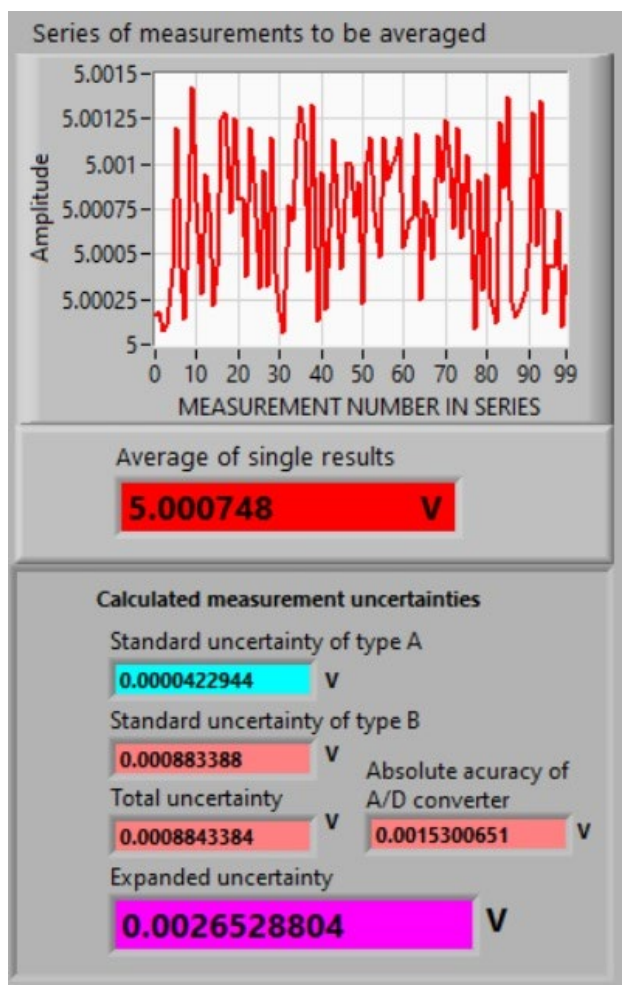

Fig.4. Series of measurements and calculated uncertainties.

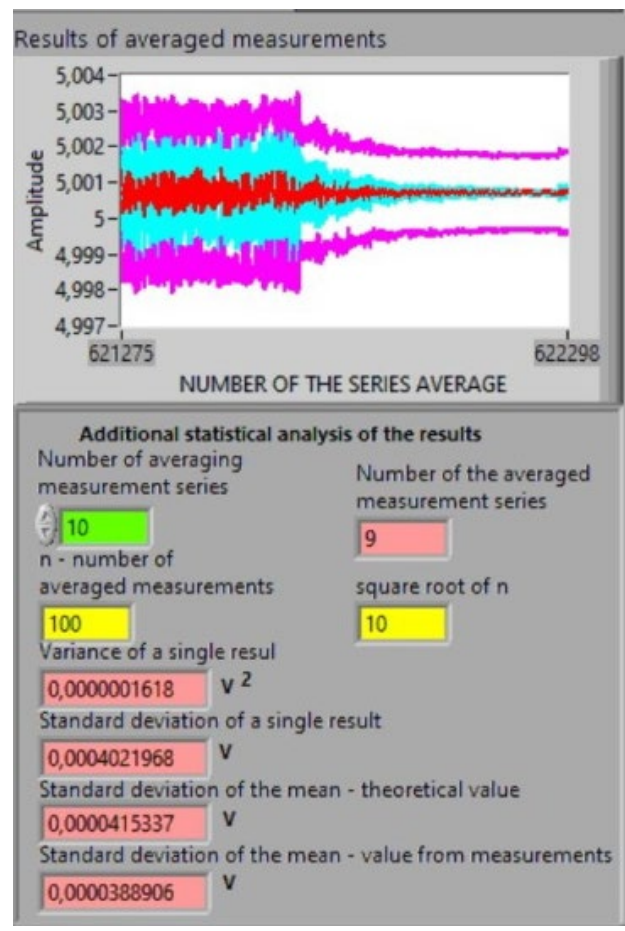

Fig.5. Additional statistical analysis of the results. 


\section{RESULTS}

Measurements were carried out with a low and a high level of interferences, each time for an increased number of measurements in the series with $n=2,5,10,20,50,100,200$, 500,1000 . For each completed series of measurements, the software calculated type A uncertainty, type B uncertainty, and expanded uncertainty $U$. The obtained results are presented in charts in Fig.6. and Fig.7. In the case of measurements with a low level of interferences (Fig.6.) and a low $n$ value, type $\mathrm{B}$ uncertainty prevails but type A uncertainty is just slightly lower and its value is similar.

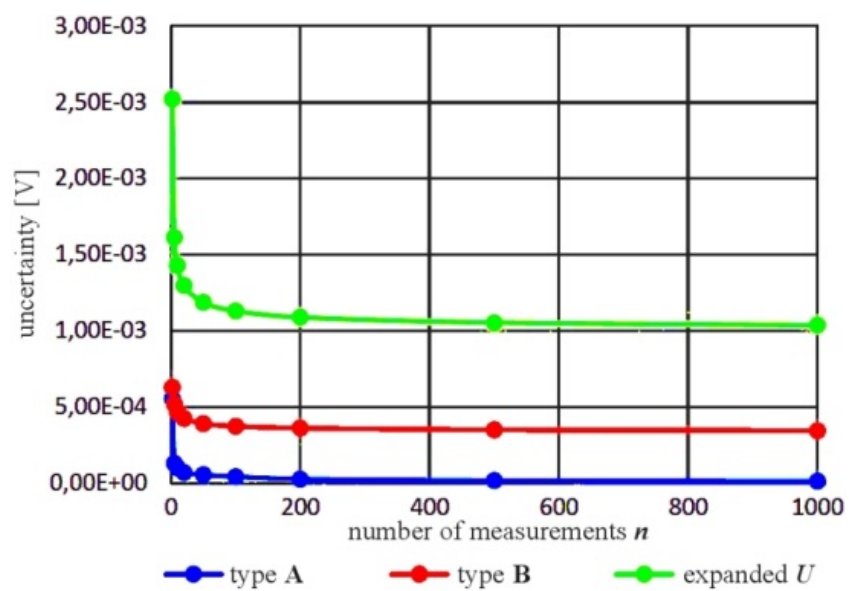

Fig.6. Type A uncertainty (blue), type B uncertainty (red) and expanded uncertainty $U$ (green) as a function of number of measurements $\mathrm{n}$ in a series for a low level of interferences

However, type A uncertainty decreases much faster in line with the increase in $n$ and constitutes merely circa $4 \%$ of type B uncertainty for $n=1000$.

It is also possible to notice that the uncertainty of type B also decreases for larger values of $n$. This may seem unclear and requires additional clarification. This is due to the fact that the type B uncertainty component related to noise is derived directly from the technical specifications, and is expressed as the RMS value divided by the square root of the number $n$ of measurements.

At the same time, however, expanded uncertainty $U$ virtually does not change already for $n \geq 200$, so series of measurements that are longer than 200 measurements do not have a metrological justification. In the case of measurements with a high level of interferences (Fig.7.), expanded uncertainty $U$ increased circa 100 -times.

Type A uncertainty prevails in these measurements and it is higher than type B uncertainty for low $n$ by two orders of magnitude. This discrepancy decreases for higher $n$ values but even for $n=1000$ type A uncertainty is higher than type B uncertainty by one order of magnitude. All uncertainties decrease in line with the increase in $n$ and even with the change from $n=500$ to 1000 expanded uncertainty $U$ decreases by another $30 \%$. However, it is impossible to achieve an uncertainty that is comparable to the measurements with a low level of interferences.

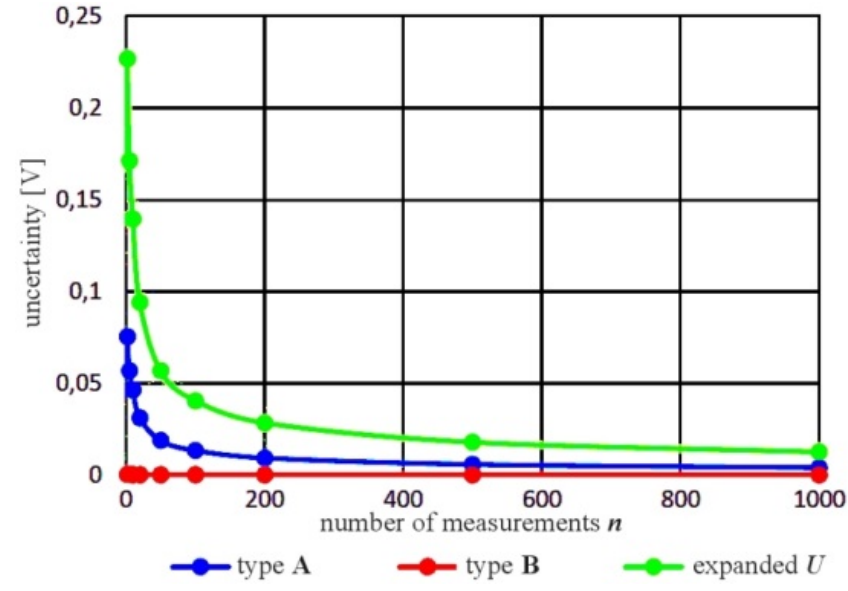

Fig.7. Type A uncertainty (blue), type B uncertainty (red) and expanded uncertainty $U$ (green) as a function of number of measurements $\mathrm{n}$ in a series for a high level of interferences.

Fig.8. shows the comparison of expanded uncertainty values $U$ for high and low level of interferences in the series with $n=2,5,10,20,50,100,200,500,1000$. For the low $n$ value expanded uncertainty $U$ in the case of high level of interferences is approximately 1000 times greater than in the case of low level of interferences. However, the difference between the two uncertainty values, for high and low level of interferences, decreases as the number of measurements $n$ increases.

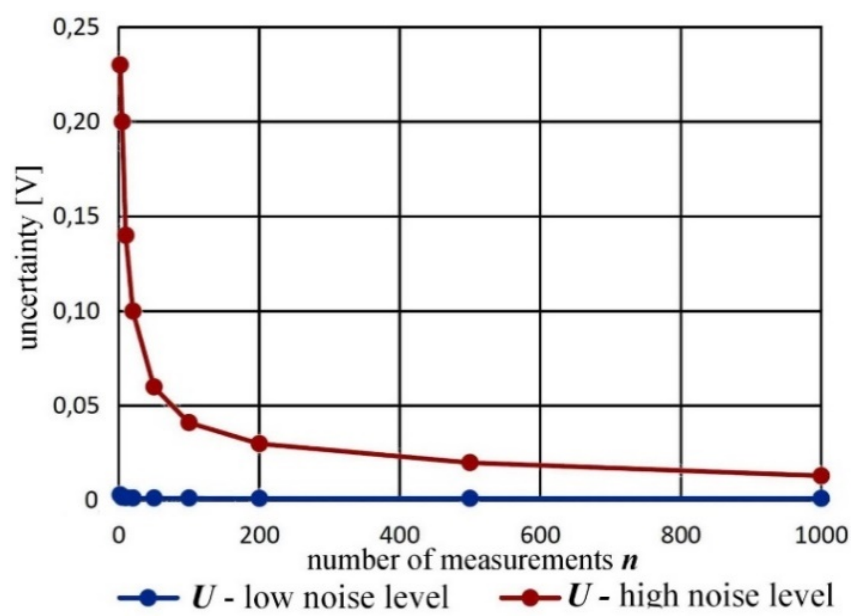

Fig.8. The comparison of uncertainty values $U$ for high and low level of interferences.

\section{CONCLUSION}

The paper presents the possibilities of utilizing the LabVIEW integrated environment in the process of evaluating the uncertainty of measurement results in direct measurement. The possibilities of presenting and visualizing the uncertainty of measurement results in a convenient and user-friendly form are discussed.

By using the LabVIEW environment - as it is illustrated with an example of the developed application - we can support or add variety to the teaching of students in the field of metrology and measurement theory. The tests reveal that 
in the case of a low level of interferences, type B uncertainty, arising from the data acquisition board accuracy, is the primary component of expanded uncertainty $U$. Type A uncertainty is considerably lower and the tests confirm that it decreases in line with the theoretical correlation in proportion to the square root of $n$ of the averaged measurements, with series longer than $n=200$ not being justifiable. In the case of a high level of interferences, type A uncertainty becomes the primary component, while type B uncertainty remains virtually at the same level. In the case of a high level of interferences, an effective way to decrease uncertainty is also to increase the number of averaged measurements, even for $n \geq 1000$.

\section{REFERENCES}

[1] Klepacki, D., Sabat, W., Kamuda, K., Kurylo, K. (2019). Signal integrity in microelectronic hybrid systems made on metal substrates. Circuit World, 45 (1), 45-51.

[2] Pawłowski, S., Plewako, J. (2015). Applicability assessment for simplified formulas to compute surface impedance at screened surfaces. Przeglad Elektrotechniczny, 12, 182-184.

[3] European Standard. (2010). Electromagnetic compatibility (EMC) Testing and measurement techniques. Power frequency magnetic field immunity test. BS EN 61000-4-8:2010.

[4] Witkovsky, V., Frollo, I. (2020). Measurement science is the science of sciences - there is no science without measurement. Measurement Science Review, 20 (1), $1-5$.

[5] Turzeniecka, D. (1999). Comments on the accuracy of some approximate methods of evaluation of expanded uncertainty. Metrologia, 36 (2), 113-116.

[6] Turzeniecka, D. (2000). Errors in the evaluation of the coverage factor as criterion of applications of approximate methods of evaluation of expanded uncertainty. Measurement, 27 (4), 223-229.

[7] Texas Instruments. (2020). REF50xx Low-Noise, Very Low Drift, Precision Voltage Reference. Data sheet SBOS410I.

[8] Joint Committee for Guides in Metrology. (2008). Evaluation of measurement data-Guide to the expression of uncertainty in measurement. JCGM 100:2008.
[9] Joint Committee for Guides in Metrology. (2012). International vocabulary of metrology - Basic and general concepts and associated terms (VIM), 3rd edition. JCGM 200:2012.

[10] European Accreditation. (2013). Evaluation of the uncertainty of measurement in calibration. EA-4/02 M:2013.

[11] Kuwałek, P., Otomański, P., Wandachowicz, K. (2020). Influence of the phenomenon of spectrum leakage on the evaluation process of metrological properties of power quality analyser. Energies, 13, 5338.

[12] Zakharov, I., Neyezhmakov, P., Botsiura, O. (2017). Verification of the indicating measuring instruments taking into account their instrumental measurement uncertainty. Measurement Science Review, 17 (6), 269-272.

[13] National Instruments. (2003). LabVIEW User Manual, Part Number 320999E-01.

[14] Wells, L.K., Travis, J. (1997). LabVIEW for Everyone. Prentice Hall PTR, ISBN 9780132681940.

[15] National Instrument. (2003). LabVIEW Measurement Manual, Part Number 322661B-01.

[16] Sokoloff, L. (2004). Applications in LabVIEW. Prentice Hall, ISBN 978-0130161949.

[17] Otomański, P., Szlachta, A. (2008). The evaluation of expanded uncertainty of measurement results in direct measurements using the LabVIEW environment. Measurement Science Review, 8 (6), 147-150.

[18] Pawłowski, E. (2017). Design and evaluation of a flowto-frequency converter circuit with thermal feedback. Measurement Science \& Technology, 28 (5), 054004054013.

[19] National Instruments. (2016). $M$ Series Data Acquisition: 16 AI, 10 DIO, 2 AO, NI 6221 (37-Pin), Device Specifications 375201C-01.

[20] Dorozhovets, M. (2019). Effectiveness of automatic correction of systematic effects in measuring chains. Measurement Science Review, 19 (4), 132-143.

Received July 09, 2021 Accepted August 25, 2021 